\title{
Adaptive Setting and Simulation of Distance Protection Relay in a Long Transmission System Connected to an Offshore Wind Farm
}

\author{
Yuan-Kang Wu, Zhou-Ting Lin, Tung-Ching Lee, Ting-Yen Hsieh, and Wei-Min Lin
}

\begin{abstract}
Offshore wind power has become the focus of the world's renewable energy development. In Taiwan, several demonstration offshore wind turbines will be scheduled to be established by 2015, and more than 300MW capacity of offshore wind farm to be installed by 2020 . As the penetration level of wind energy increases, more reliable protection systems for offshore wind farms are required. The wind farm protection system is usually divided into different protection zones including the wind farm area, wind farm collection system, wind farm interconnection system and the utility area. Different protective zones have different protection schemes and relay settings. This project has proposed an adaptive distance relay that protects submarine cables between an offshore wind farm and an AC grid. The double-circuit submarine cables that connect offshore wind farm in Penghu and Taiwan grid will be utilized as an example. First, the apparent impedance of the relay is mathematically derived using a detailed equivalent circuit model, and the characteristic trip boundaries of the relay in various scenarios are obtained. The nonlinear characteristics of the trip boundaries that are used in a typical distance relay with four protection zones will be discussed. Furthermore, the effects of the uncontrollable factors on the performance of a distance relay have been considered into the simulations. These system disturbances include wind speed variation, system short circuit faults, or system component failures. These case studies have been presented using the simulation package to evaluate the feasibility of the distance relay setting that is developed in this project.
\end{abstract}

Index Terms-Offshore wind power, protection system, distance relay.

\section{INTRODUCTION}

Taiwan is a highly energy dependent country that has followed the world trend in developing renewable energy. In order to cooperate to achieve the goal of green power policy proposed by the government as soon as possible, and meet the developing requirement of framework on climate change in the future, Taiwan Power Company (TPC) in 2002 sketched a developing plan of wind power generation for ten years as the

Manuscript received July 9, 2015; revised October 9, 2015. This work was supported by the Ministry of Science and Technology (MOST) of Taiwan under Grant 104-3113-E-194 -001 -CC2. Project title: Development of Integration Technology for Large-Scale Offshore Wind Farms in Taiwan (2/3) and by the Ministry of Economic Affairs of Taiwan under Project: The Proposal for the Photovoltaic Environment Construction and Industry Promotion (Project number: 104-D0304).

Yuan-Kang Wu and Zhou-Ting Lin are with the National Chung-Cheng University, Chiayi, 62102, Taiwan (e-mail: allenwu@ccu.edu.tw).

Tung-Ching Lee, Ting-Yen Hsieh, and Wei-Min Lin are with Green Energy and Environment Research Laboratories, Industrial Technology Research Institute, Hsinchu, 31040, Taiwan. impetus of application in wind power generation. In addition, in order to promote sustainable energy development continually, Bureau of Energy (BOE) of the Ministry of Economic Affairs (MOEA) inaugurated the Offices of Million Solar Rooftop PVs and Thousand Wind Turbines Promotion in 2012. The key tasks of "Thousand Wind Turbines Promotion" are identifying regulatory hindrances and proposing possible resolutions, executing offshore wind power demonstration projects, and helping the development of domestic wind power industry to fulfill the target of constructing 450 onshore wind turbines by 2020 and 600 offshore wind turbines by 2030. In fact, offshore wind power has become the focus of the world's renewable energy development. With the gradually maturing of offshore wind power technology, the power cost and operation cost of offshore wind power are gradually reduced. Therefore, by 2012, the total installed capacity all over the world has been reached up to $3813 \mathrm{GW}$, accounting for about $9 \%$ of the European market, with around 28 offshore wind farms located in Denmark, UK, Ireland, Sweden, Netherlands and Germany. The European Wind Energy Association (EWEA) estimates that by 2020, $40 \mathrm{GW}$ of offshore wind power will produce 148 TWh annually, meeting over $4 \%$ of the EU's total electricity demand and avoiding 87 million tonnes of $\mathrm{CO}_{2}$ emissions.

The system framework comprises Penghu offshore wind farm, Penghu grid, Taiwan grid and $58.9 \mathrm{~km}$ submarine cable connecting Taiwan and Penghu grid. The scheme diagram of the studied system framework is shown in Fig. 1.

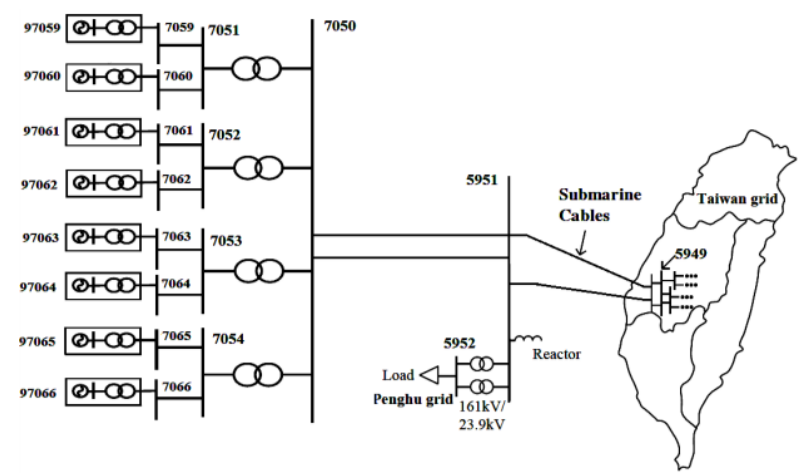

Fig. 1. Scheme diagram of system framework.

Many research works have studied the use of protection relays in wind farms [1]-[9]. Conventional methods to protect an individual wind turbine generator were proposed in [7], [8]. These methods use over/under voltage, over/under frequency, instantaneous phase/neutral over-current for generator phase/ground faults, and inverse time phase over-current for 
generator overload. They can successfully protect a wind turbine when an internal fault occurs. The proper delayed operation time of an over-current relay that is used in neighboring distribution feeders that are connected to a wind farm interconnected has been studied [2] by undertaking a case study. In that study, the simulation considered the various fault conditions and the power output of the wind farm demonstrated that the setting of the optimal operating time of the over-current relay could improve the operational reliability and stability of the wind farm. The issue of the undesirable operation of zone 3 impedance relays owing to induction generator interconnections has been studied [4]. In that study, the static characteristics of an induction generator were derived, and a sensitivity model of the operation margin of the impedance relays was proposed to evaluate the relay performance against undesirable operation in a stressed system. Reference [5] described a new type of relay suitable for the protection of wind farms. The relay, installed at the utility end of a power collection circuit, is designed to protect the collection circuit and the LV and MV windings of each wind turbine generator transformer. The relay has been tested on-site at the Cemmaes wind farm. The main purpose of the trial was to confirm that the relay remained stable during all types of disturbances that originate from the utility network. These mainly consisted of voltage clips, momentary interruptions, sustained under-voltages and occasionally loss of mains.

In order to minimize the outage zone in a wind farm, a protection relay for a wind turbine generator based on positive- and negative-sequence fault components is proposed in [6]. Reference [9] also proposed a directional-relay algorithm based on positive sequence fault components that have inherent advantages in identifying fault direction. Other important studies of wind farm protection are shown in [10]-[13]. In [10], the design and evaluation of a protection relay for a wind farm with fixed-speed induction generators were proposed. Reference [12] discusses protective relaying for large wind generation plant collector systems; several important protections in a wind farm was discussed, including transmission line protection, collection station transformer protection, collector feeder protection, substation power factor correction capacitor protection and dynamic VAR system feeder protection. Reference [13] investigates the effects of wind turbines on the new optimal coordination of directional over-current relays in power systems, in which the different effects of over-current relays are considered and a systematic approach to include their effects is illustrated. The results in [13] show that the wind turbines have effects on the optimal time settings. The distance relay is a sort of relay that detects fault distance, i.e. whether the impedance of distance between the relay and fault point is lower than the set value of relay, which judges whether the fault occurred in the protective range of the relay. The basic principle of distance relay is Ohm's law; thus, the distance relay must have the current and voltage signal inputs of the system. The distance between the relay and the fault point can be measured according to fault current $I_{F}$ and system voltage drop $V_{F}$, as resulted from the fault. If the preset protective range of relay is $Z>Z_{F}$, the relay then identifies the internal fault and operates. Fig. 2 shows the protection coordination of distance relay.
The main protection is assumed to be located in the $A B$ section; thus, if the fault point is in this range, the relay quickly trips. The farther the distance, the longer the actuation time required by the relay; thus, the coordination time of backup and main protection shall be controlled in order to avoid relay misoperation.

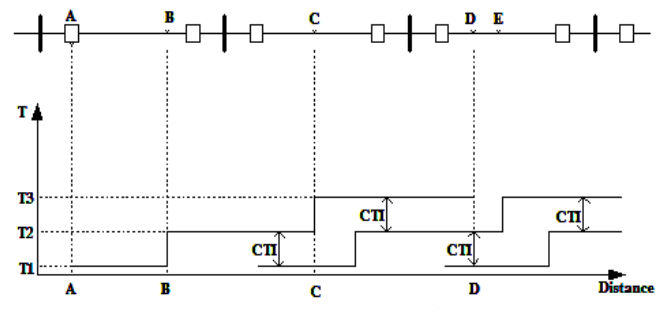

Fig. 2. Protection coordination of distance relay.

The distance relay is mostly used for cable protection in the long range. Therefore, distance relay is often used for protecting the transmission line between a wind farm and a substation. As wind force changes very quickly, the voltage and current shall be instantly monitored, and the tripping time of relay must consider protection coordination in the fault state. The distance relay detects fault voltage $\mathrm{V}$ and fault current I, and obtains fault impedance by Ohm's law. There are many probable factors influencing the three parameters, and the protective range of the relay can be observed by changing specific variables.

\section{DESIGN OF Distance RELAY}

Fig. 3 presents the protection zone of the polygonal distance relay that was used in several systems. Traditionally, the settings of trip boundaries are based mainly on the percentage of the line impedance. For current pilot protection setting of transmission line in Taiwan, the zone 1 is customarily set between $80 \%$ and $85 \%$ line impedance of the main protected line, and if the trajectory of measured impedance swings into this zone, the circuit breaker trips with no intentional time delay. The zone 2 , also called time delayed protection zone, covers zone1 and overreaches to the part of the next line section; it is set to the main protected line impedance plus $50 \%$ of the shortest line emanating from the remote bus, still, its operating time is 20 cycle time to coordinate with zone 1 protection. The zone 3 is used to provide remote backup protection to isolate the fault in the event that the remote circuit breaker fails to trip; it is set at the summation of main line length, $100 \%$ of the longest next line section, and $25 \%$ of the shortest line length after next, also, the tripping time is of the order of 30 cycle time. Finally, the zone 4 could provide further back-up protection, and safeguard the transmission line behind the forward protection zone. In addition, to manage the circumstance wind turbine trips prior to zone operation when fault occurs on submarine cable, introduction of intelligent tripping control procedure dedicated to submarine cable protection is of concern for improving performance of proposed polygonal distance relay.

Fig. 4 presents the protection zone of the quadrilateral distance relay that was used in this project. It clearly demonstrates that the trip boundary of a typical quadrilateral distance relay is characterized by four straight lines. In this 
project, the ideal trip characteristic will be analyzed by using the power system simulation. Then, the problems that are encountered can be discussed, and their solutions can be subsequently proposed.

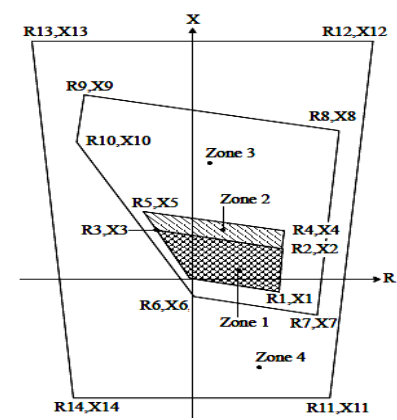

Fig. 3. Typical scheme of a four stage polygonal distance relay.

\section{A. Specification of Characteristic Boundaries}

The characteristic boundaries of installation capacity 200MW with different power transfer angle and voltage ratio are shown in Fig. 4, which consist of four boundary lines shown for $Z_{F}(0-25 \Omega)$ and fault location (10-90\%); those are described below:

Line I: solid faults at different locations;

Line II: faults at a relay-reach end ( $90 \%$ of line length) with different fault resistance up to $25 \Omega$;

Line III: faults at different points with $25 \Omega$ fault resistance;

Line IV: faults at the relaying point with different fault resistance up to $25 \Omega$.

Apart from line I, line II to IV changes with the operating conditions of the wind farm. The approach to making distance relay in a manner that is sensitive to such conditions has been discussed and two approaches have been proposed to solve this problem. Firstly, setting patterns of the relay operation under all possible system conditions are calculated off-line and stored in a data table for indexing during faults. Secondly, only one set of parameters set in all instances are calculated on-line and these are updated from time to time as the conditions of the system change beyond a specified limit. When a fault occurs, the relay operates with the latest setting parameters, which defines an optimal characteristic boundary. Of these two approaches, the former requires a large amount of computer hard-disc storage to store the data and a memory to ensure the parameters are set precisely; the latter may require a CPU and memory that provide a faster processing speed.

\section{B. Trip Boundary Planning Considering System Operation Status}

The following subsection will discuss the setting of each trip boundary of proposed distance relay zones with polygonal characteristic for submarine cable protection shown in Fig. 4.

The setting of point $\mathrm{A}$ in zone 1 is chosen to meets the current regulations of relay setting in Taiwan and accords with actual system operation. The real part of point $\mathrm{A}$ is slightly larger than zero and its included angle with reactance axis is approximately 85 degree, i.e. impedance angle of transmission line. The border from origin terminating on point $\mathrm{B}$ is chosen to ensure the huge system disturbance with capacitive characteristic could nearly be encompassed by zone 1 element. Hence, the designing reflects tendency towards fourth quadrant, that is, load with leading power factor. The straight line through point $\mathrm{A}$ terminates on point $\mathrm{C}$. For one thing, it guarantees the tripping protection zone with instantaneity and high speed operating characteristics when fault occurs on the threshold point of zone 1 setting with the increasing of earth fault impedance. For another, the zone planning takes the huge disturbance with inductive characteristic into account. Moreover, compared with conventional scheme, proposed four stages polygonal distance relay is provided with flexible impedance coverage, especially the resistive reach settings that is independently adjustable to fulfill the current system operation status, overcome high value of fault resistance and provide a sophisticated protection for submarine cable. Consequently, the origin and points $\mathrm{A}$ to $\mathrm{C}$ form the trip boundary of relay zone 1.

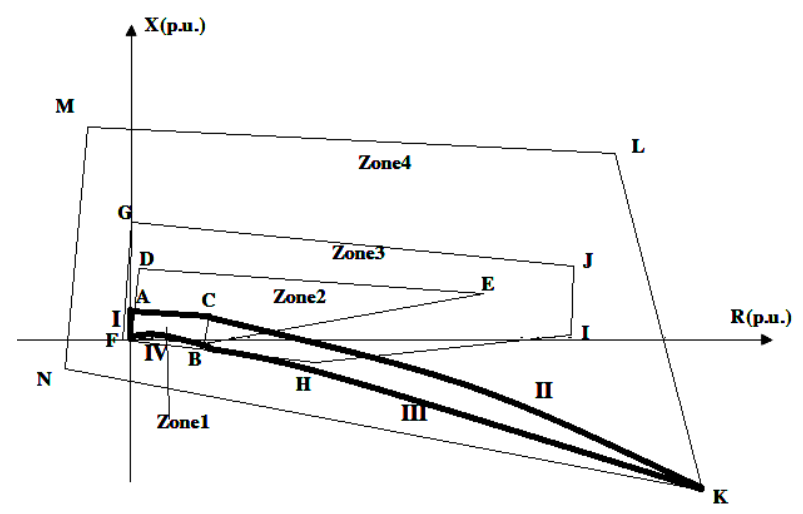

Fig. 4. An ideal trip boundary under given system conditions.

A straight line connecting with points $\mathrm{D}$ and $\mathrm{E}$ is of the essence to back up zone 1 setting for the failure of blocking grounded fault on submarine cable. The border of zone 2 starts from point B and extends to point E does not merely extend to the farthest resistive reach of stage 1 but go a step further for overreaching to the next line section emanating from remote bus. The protective coordination with previous stage is considered. Also, zone 2 setting could provide fault defense of entire cable. The trip boundary of zone 2 is constituted by origin and points $\mathrm{B}$ to $\mathrm{E}$.

The following two stage setting of proposed scheme is of concern as the lager tripping boundary is designed to offer a more comprehensive protection. Power swings, load variation, and small signal instability in the power system may contribute to the undesirable operation of relay caused by the incorrect settings of zone 3 and zone 4 elements. The phenomenon is obvious providing the aforesaid state of affairs take place on submarine cable or connection points.

The operating region of zone 3 element consists of five borders. The situation may come about when the wind farm produces a little or zero power and fails to supply steady electric power to load demand of Penghu archipelagoes; as a result, the active power flow on submarine cables is reversely transmitted from Taiwan grid to Penghu. If a severe grounded fault occurs on submarine cable during this time, the impedance trajectories does swing to left half-plane. Hence, blocking should be enforced with intentional delay by another 
relay that senses the reverse power flow from Taiwan to Penghu.

Zone 4 is designed to prevent the protection maloperating during the start-up of a wind turbine when there is a little or no active generation on the collection circuit. During start-up, a generator may be motored up to speed and consequently demands both active and reactive power from the network. If the WTGs already connected to the collection circuit are not generating or no WTGs are connected, motoring causes the impedance curve enters into the protection zone. It provides the wide protection to reverse power transmission, mainly aims at protection of submarine cable linking Penghu archipelagoes and Taiwan grid.

\section{Simulation Results}

The simulation system framework of this study consists of four regions: Penghu offshore wind farm, Penghu power grid, Taiwan power grid, and the $58.9 \mathrm{~km}$ submarine cable connecting Taiwan to Penghu. In this study, the total installed capacity of the Penghu offshore wind farm is assumed to be 200MW, with 8 sets of wind turbines, and each set has 7 wind turbine units. For each set, the full-load output active power is $25 \mathrm{MW}$. The system one-line diagram is as shown in Fig. 1, where bus 5951 is the wind farm PCC point, bus 5952 is the equivalent load bus in the Penghu area, and bus 5949 is the connection point of the submarine cable at the Taiwan grid side.

\section{A. Simulation Analysis 1: Influence of Wind Gust up on Impedance Detected by Distance Relay}

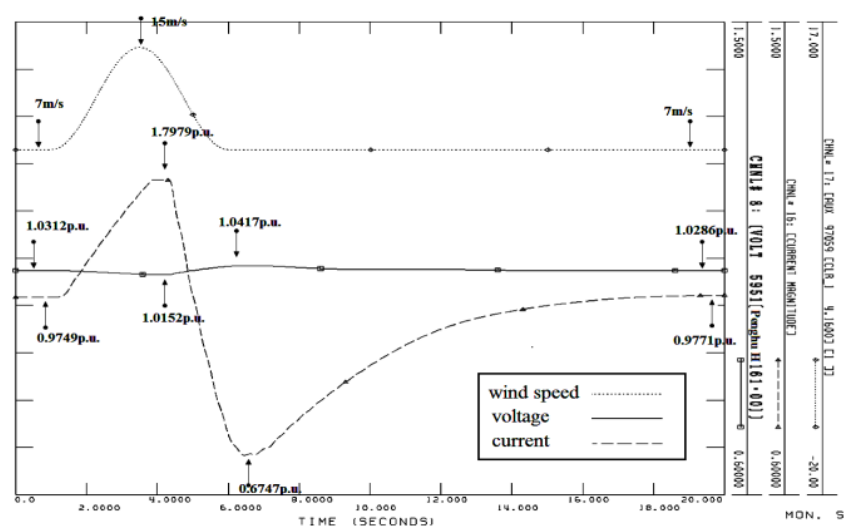

Fig. 5. The voltage and current waveforms detected by distance relay at the wind farm PCC.

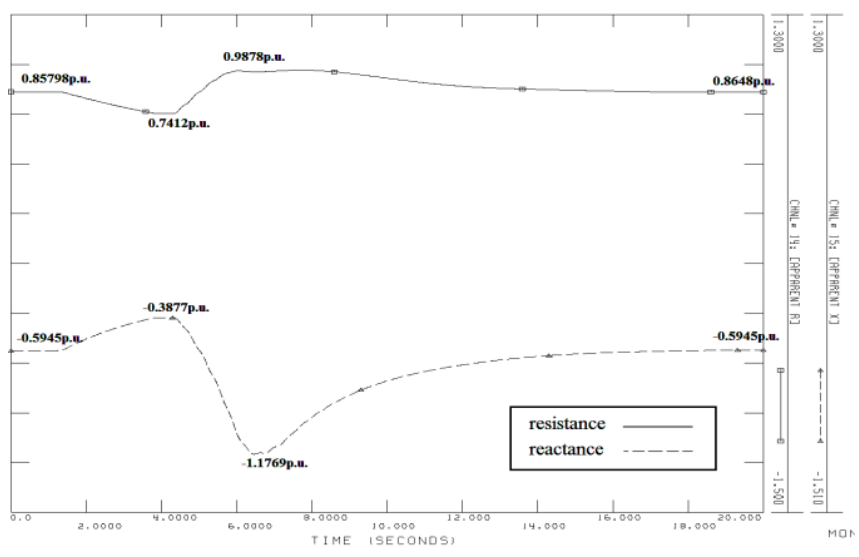

(a)

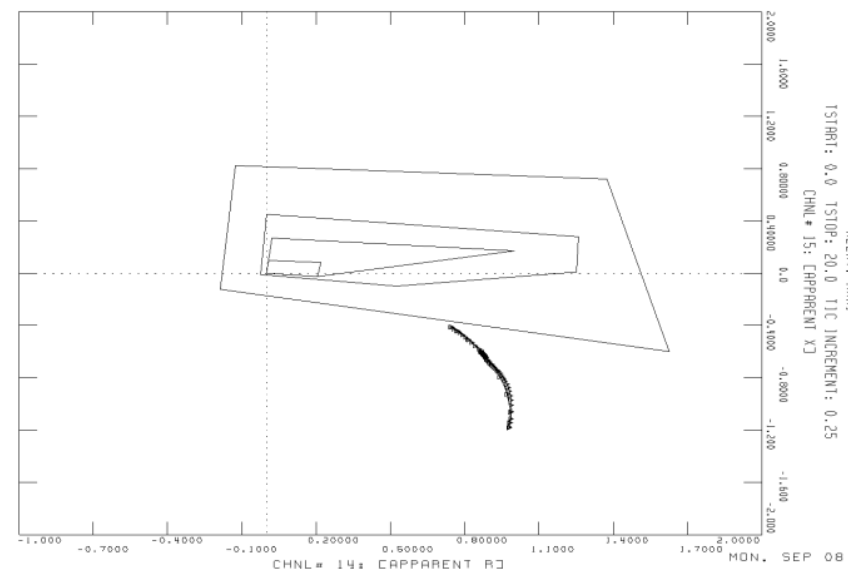

(b)

Fig. 6. Sequence (a) and R-X impedance (b) diagram with a protective area of relay.

In this case, if the wind gust up occurs at $1 \mathrm{~s}$ : the initial wind speed is $7 \mathrm{~m} / \mathrm{s}$, and maximum wind speed is $15 \mathrm{~m} / \mathrm{s}$, which is maintained for $5 \mathrm{~s}$. The voltage and current waveforms, as detected by distance relay at the wind farm connection point, are shown in Fig. 5, where the solid line is the voltage waveform, the dotted line is the current waveform, and the unit of voltage and current are p.u. value. It is observed that the voltage variation has little change; whereas, the current increases with the wind turbine output active power, and the current value decreases with the wind speed. In this case, the frequency increases with the wind speed; and the frequency decreases with the wind speed. However, the range of frequency change is between $60.01 \mathrm{~Hz}$ and $59.983 \mathrm{~Hz}$, which is the normal frequency operating range of the system. The current increases with the wind speed; however, the voltage fluctuates slightly, indicating a decrease in equivalent impedance. In other words, the absolute values of resistance and reactance decrease, as shown in Fig. 6. On the contrary, when the current decreases with wind speed, the equivalent impedance increases. The resistance and reactance sequence diagram of Fig. 6 (a) is plotted into an R-X impedance diagram, and added with a protective area of relay. The result is shown in Fig. 6 (b). It is observed that, while the change in wind speed results in wind farm system transient, the distance relay will not be actuated falsely under the effect.

\section{B. Simulation Analysis 2: Influence of Wind Ramp up on Impedance Detected by Distance Relay}

In this case, the initial wind speed is $7 \mathrm{~m} / \mathrm{s}$, the wind speed increases to $15 \mathrm{~m} / \mathrm{s}$ within $5 \mathrm{~s}$. The voltage and current waveforms, as detected by distance relay at the wind farm connection point, are as shown in Fig. 7, where the solid line is the voltage waveform, and the dotted line is the current waveform. It is observed that the voltage variation almost remains the same, and the current increases with the initial wind turbine output active power. However, when the wind speed is maximized at $15 \mathrm{~m} / \mathrm{s}$ and stabilized, the current decreases and restores to the original steady-state current. The initial frequency value of this system is $60.00 \mathrm{~Hz}$, and when wind speed rises suddenly at $1 \mathrm{~s}$, the system frequency increases accordingly to its maximum of $60.01 \mathrm{~Hz}$. However, as the wind speed is stable, the frequency gradually returns to the initial value $60 \mathrm{~Hz}$. 
The current increases with the wind speed; however, the voltage fluctuates slightly, indicating a decrease in impedance. In other words, the absolute values of resistance and reactance decrease, as shown in Fig. 8. However, when the wind speed reaches maximum $15 \mathrm{~m} / \mathrm{s}$ and is stable, the current falls, and the voltage changes slightly.

The resistance and reactance sequence diagram of Fig. 8 (a) is plotted into an R-X impedance diagram, and added with a protective area of the relay. The result is shown in Fig. 8 (b). It is observed that the change in wind speed results in wind farm system transient, the distance relay will not be actuated falsely under the effect.

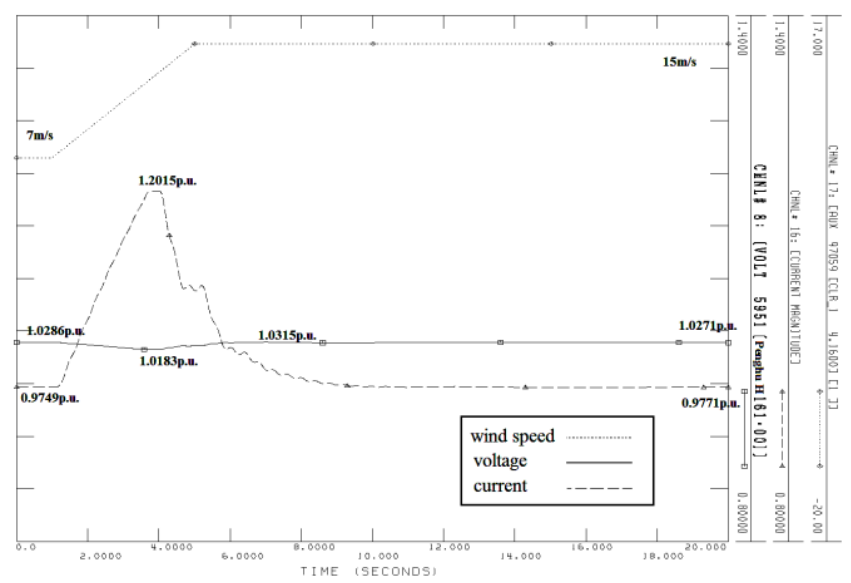

Fig. 7. The voltage and current waveforms detected by distance relay at the wind farm PCC.

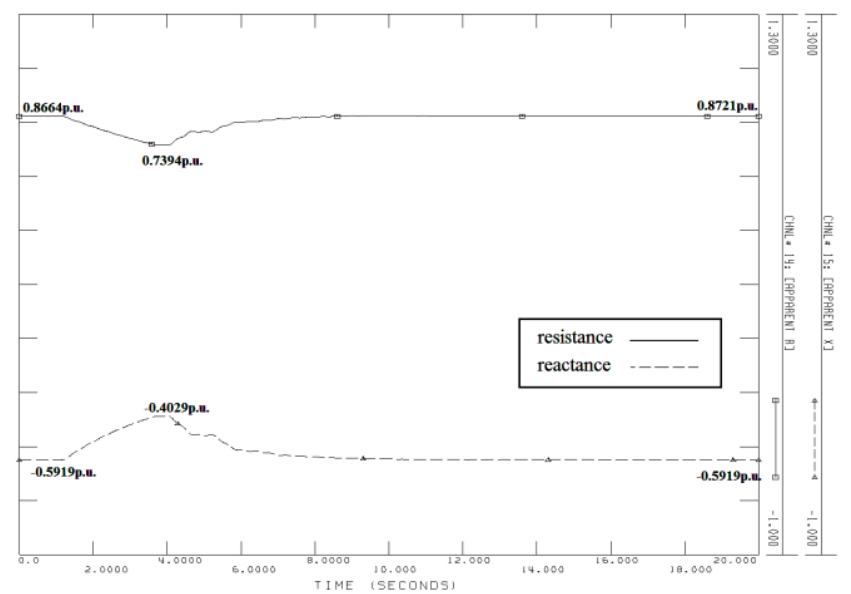

(a)

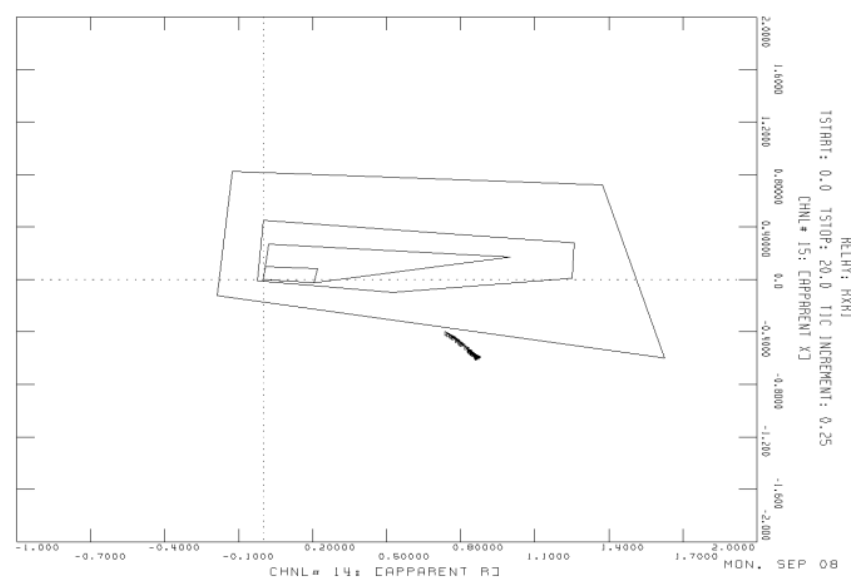

(b)

Fig. 8. Sequence (a) and R-X impedance (b) diagram with a protective area of relay.

\section{Simulation Analysis 3: Influence of Three-Phase Short-Circuit Ground Fault on Impedance Detected by Distance Relay}

This case simulates a three-phase short-circuit ground fault in the output of a group of wind turbines. In this case, the voltage at the wind farm connection point suddenly drops to $0.7901 \mathrm{pu}$, and the fault current is $1.8791 \mathrm{pu}$, as shown in Fig. 9. Therefore, the absolute value of instantaneous impedance measured by distance relay decreases. The resistance and reactance sequence diagram in Fig. 10 is plotted into an R-X impedance diagram, and added with a protective area of relay. It is observed that the distance relay will not be falsely actuated when this short circuit accident occurs.

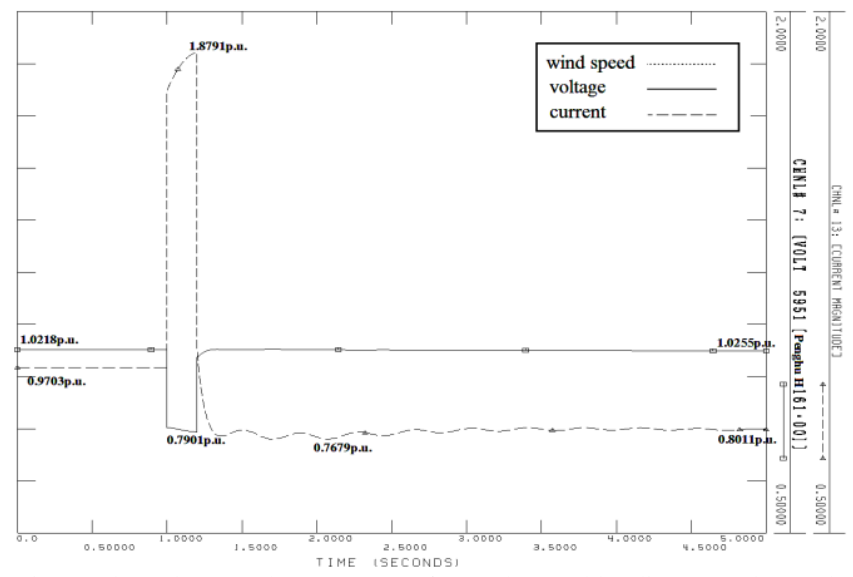

Fig. 9. The voltage and current waveforms detected by distance relay at the wind farm PCC.

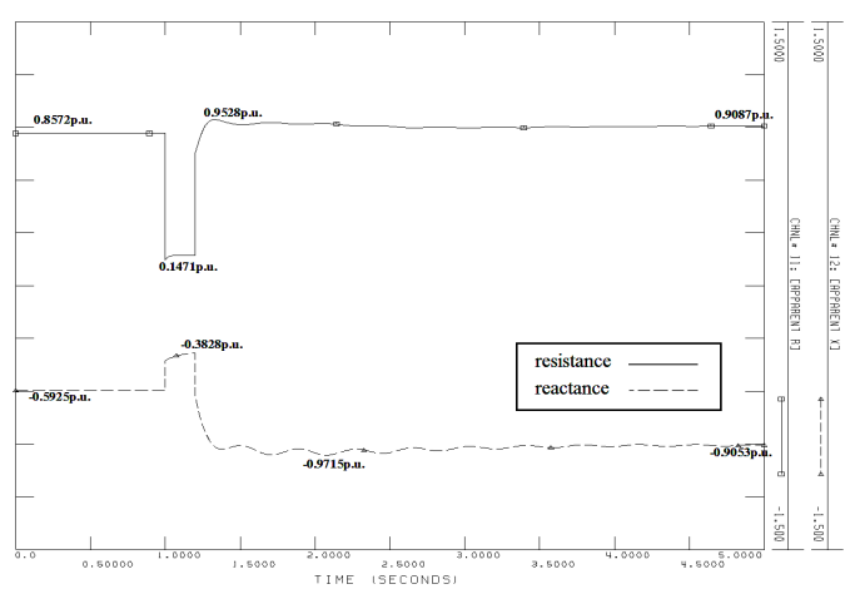

(a)

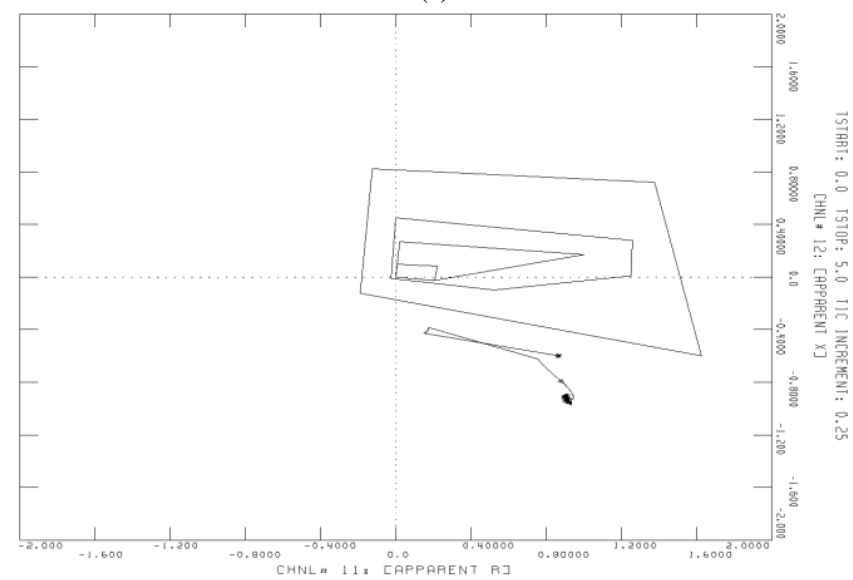

(b)

Fig. 10. Sequence (a) and R-X impedance (b) diagram with a protective area of relay. 


\section{Simulation Analysis 4: Influence of the Fault of a Submarine Cable on Impedance Detected by Distance Relay}

This case assumes that the three-phase short-circuit fault occurs in one cable of a two-circuit submarine cable, and then the impedance changes, as detected by distance relay at the wind farm connection point, and the voltage and frequency variations in the buses of both sides of the submarine cable are observed. Fig. 11 shows the voltage and current waveforms detected by relay during grid fault. It is observed that the voltage instantly drops to 0.0069 pu during fault; whereas, the current rises instantly. Therefore, the absolute value of instantaneous impedance measured by distance relay decreases instantly. The initial frequency value of this system is $60.00 \mathrm{~Hz}$, and during the fault, frequency increases accordingly to its maximum of $60.54 \mathrm{~Hz}$.

The resistance and reactance sequence diagram measured in Fig. 12 (a) is plotted into an R-X impedance diagram, and added with a protective area of relay. The result is shown in Fig. 12 (b). According to this figure, the impedance is in the quadrant four in a steady state at $1 \mathrm{~s}$. It moves towards the zero point after the fault occurs, and the protective range of the relay is touched; thus, the relay trips. The impedance moves towards the quadrant three after the fault is cleared. At this point, the power flow direction is changed, and the impedance direction detected by relay changes accordingly.

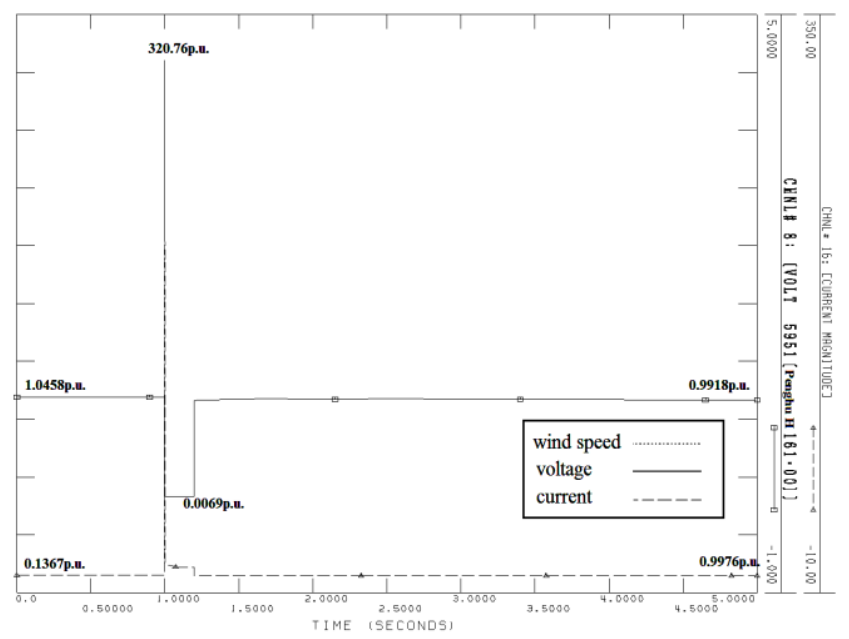

Fig. 11. The voltage and current waveforms detected by distance relay at the wind farm PCC.

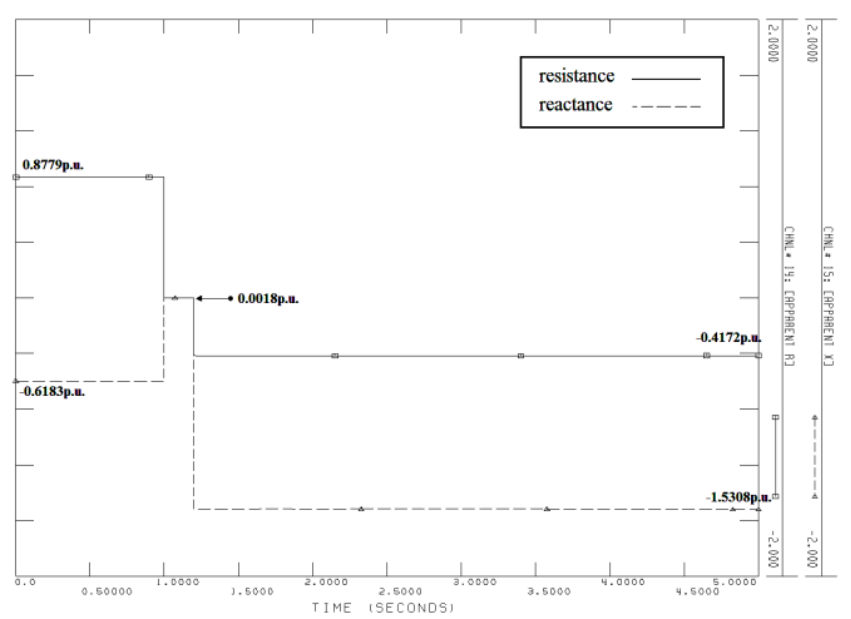

(a)

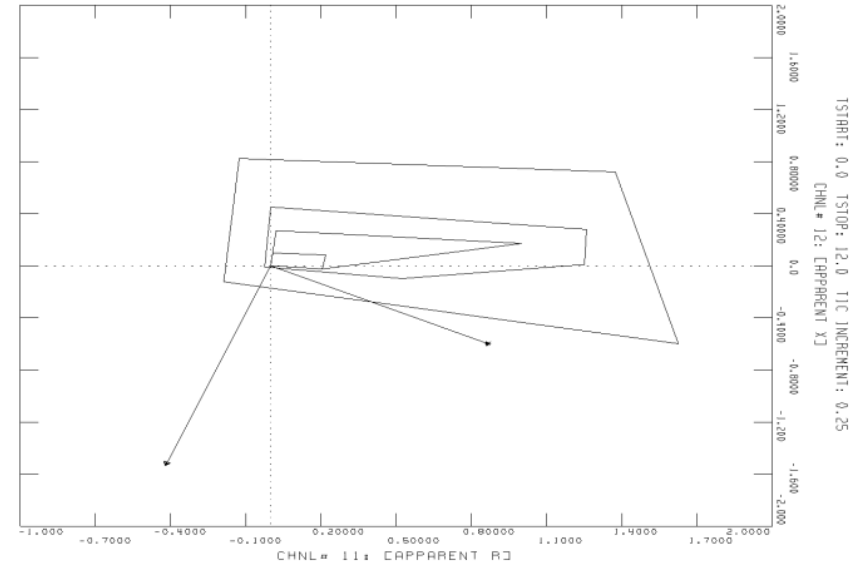

(b)

Fig. 12. Sequence (a) and R-X impedance (b) diagram with a protective area of relay.

E. Simulation Analysis 5: Influence of the Fault of a Point-of-Common Coupling (PCC) on Impedance Detected by Distance Relay

This case assumes that the three-phase short-circuit fault occurs in PCC, and then the impedance changes, as detected by distance relay at the wind farm connection point, and the voltage and frequency variations in the buses of both sides of the submarine cable are observed. Fig. 13 shows the voltage and current waveforms detected by relay during grid fault. It is observed that the voltage instantly drops to $0.0109 \mathrm{pu}$ during fault; whereas, the current rises instantly. Therefore, the absolute value of instantaneous impedance measured by distance relay decreases instantly. Fig. 13 shows the transient frequency curve during the fault. During the fault, frequency increases accordingly to its maximum of $60.54 \mathrm{~Hz}$.

The resistance and reactance sequence diagram measured in Fig. 14 (a) is plotted into an R-X impedance diagram, and added with a protective area of relay. The result is shown in Fig. 14 (b). According to this figure, the impedance is in the quadrant four in a steady state at $1 \mathrm{~s}$. It moves towards the zero point after the fault occurs, and the protective range of the relay is touched; thus, the relay trips. The impedance moves towards the quadrant three after the fault is cleared. At this point, the power flow direction is changed, and the impedance direction detected by relay changes accordingly.

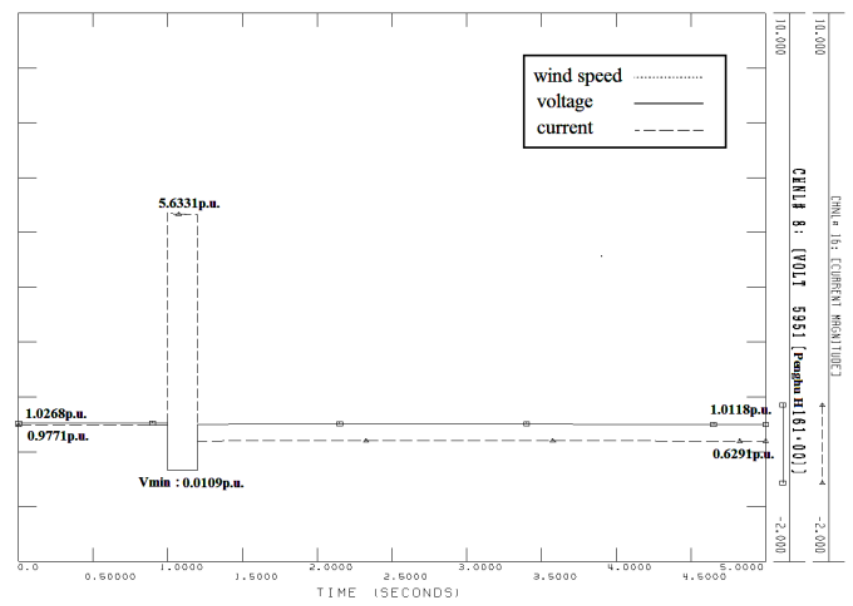

Fig. 13. The voltage and current waveforms detected by distance relay at the wind farm PCC. 


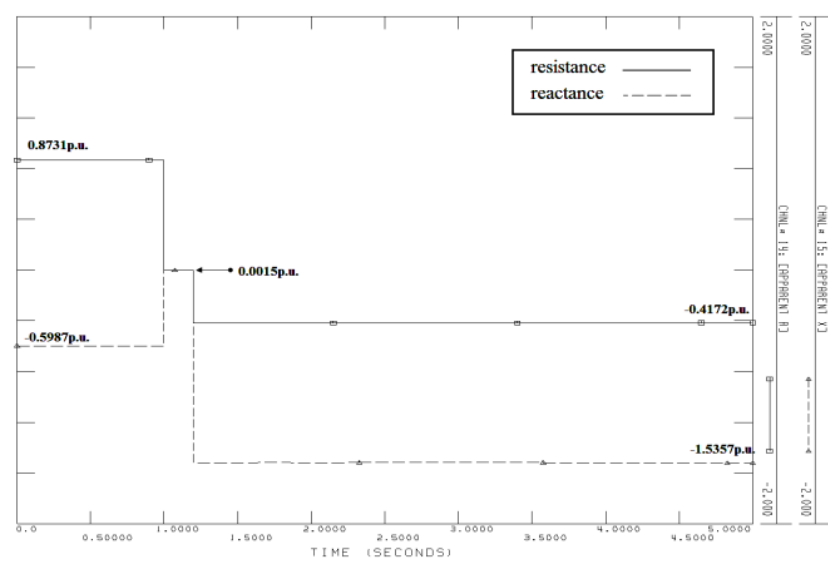

(a)

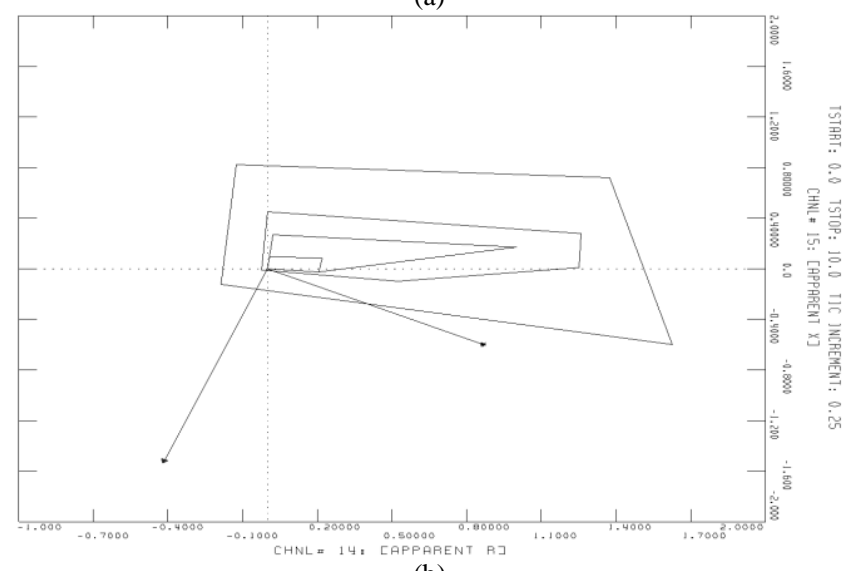

(b)

Fig. 14. Sequence (a) and R-X impedance (b) diagram with a protective area of relay.

\section{CONCLUSION}

This study uses PSS/E to simulate the parallel connection of the Penghu offshore wind farm to the Penghu system. Moreover, it analyzes the transient of the parallel connection of the long-distance submarine cable to Taiwan's power grid, and discusses the actuation of distance relay at the wind farm connection point under different system interferences or accidents. According to the simulation results, when a three-phase ground fault occurs in the wind farm side, meaning the wind turbine output to the PCC of wind farm, the distance relay can detect transient impedance changes. However, the detected impedance will not touch the protective range of distance relay, as the relay setting has considered directionality. Comparatively, when one cable of a two-circuit submarine cable has a three-phase short-circuit fault, as the two-circuit submarine cable is the main range protected by distance relay, the fault impedance detected by the relay will touch the protective range, and the protective relay is actuated and cut off. In addition to the distance relay at the wind farm connection point, the wind turbine set has undervoltage and underfrequency protection relays. In the case of actuated distance relay, the distance relay is actuated prior to the undervoltage and underfrequency protection relays for wind turbines. In other words, this distance relay can operate normally when the system is in transient.

\section{REFERENCES}

[1] A. K. Pradhan and G. Joós, "Adaptive distance relay setting for lines connecting wind farm," IEEE Trans. Energy Conversion, vol. 22, no. 1, pp. 206-213, March 2007.
[2] S. I. Jang, J. H. Choi, J. W. Kim, D. M. Choi, and K. H. Kim, “An adaptive relaying for the protection of a wind farm interconnected with distribution networks," IEEE PES Transmission and Distribution Conference and Exposition, vol. 1, pp. 296-302, 2003.

[3] N. Acharya and C. C. Liu, "Tripping of wind turbines during a system fault," in Proc. IEEE Power and Energy Society General Meeting Conversion and Delivery of Electrical Energy in the 21st Century, 2008, pp. $1-8$

[4] S. Li, S. Sun, and S. Li, "Operation characteristics of zone 3 impedance relays in wind power systems with fixed - speed induction generators," in Proc. Power and Energy Engineering Conference, 2011, pp. 1-6.

[5] P. Crossley, S. Haslam, and N. Jenkins, "Evaluation of a new type of protection relay for wind farms," in Proc. IEE Colloquium on System Implications of Embedded Generation and Its Protection and Control, 1998.

[6] T.-Y. Zheng, S.-T. Cha, P. A. Crossley, and Y.-C. Kang, "Protection algorithm for a wind turbine generator based on positive- and negative-sequence fault components," in Proc. International Conference on Advanced Power System Automation and Protection, 2011, vol. 2, pp. 1115-1120.

[7] GE Consumer \& Industrial Multilin, W650-Wind Generator Protection System Instruction Manuals, 2006 GE Multilin.

[8] Schweitzer Engineering Laboratories, SEL-700GW Wind Generator Relay.

[9] H. Gao and P. A. Crossley, "Design and evaluation of a directional algorithm for transmission-line protection based on positivesequence fault components," IEE Proceedings - Generation, Transmission and Distribution, vol. 153, issue 6, pp. 711-718, 2006.

[10] S. J. Haslam, P. A. Crossley, and N. Jenkins, "Design and evaluation of a wind farm protection relay," IEE Generation, Transmission and Distribution, vol. 146, no. 1, pp. 37-44, January 1999.

[11] S. M. Brahma and A. A. Girgis, "Development of adaptive protection scheme for distribution systems with high penetration of distributed generation," IEEE Trans. Power Delivery, vol. 19, no. 1, pp. 56-63, January 2004.

[12] L. L. Henriksen and J. J. Kumm, "Protective relaying applied to large wind plant collector systems," in Proc. IEEE/PES Transmission and Distribution Conference and Exposition, 2008.

[13] D. Jones and K. Bennett, "Wind farm collector protection using directional over-current elements," in Proc. IEEE/PES Transmission and Distribution Conference and Exposition, 2012.

Yuan-Kang Wu received the Ph.D. degree in electronic and electrical engineering from the University of Strathclyde, Glasgow, U.K., in 2004. He was a researcher at the Industrial Technology Research Institute, Hsinchu, Taiwan, and an engineer at the Taiwan Electric Research and Testing Center, Taiwan. He is currently an associate professor in the Department of Electrical Engineering, National Chung-Cheng University, Chiayi, Taiwan, working in the area of wind turbine modeling, wind power systems, offshore wind farm planning, renewable energy forecasting techniques, power system control and management, distributed generation, and smart grid control.

Zhou-Ting Lin received the M.S. degree in the Department of Electrical Engineering from National Chung Cheng University, Chiayi, Taiwan, in 2015. He will work at Taiwan Power Company from 2015.

Dong-Jing Lee received his Ph.D. degree from the Department of Electrical Engineering, National Cheng Kung University, Tainan, Taiwan, in June 2009. He is a senior research in Green Energy Department of Industrial Technology Research Institute. His interests are power system dynamics and renewable energy.

Ting-Yen Hsieh received his Ph.D. degree in electrical engineering from National Taiwan University of Science and Technology, Taipei, Taiwan in 2013. He is currently a researcher in the Industrial Technology Research Institute, Hsinchu, Taiwan. His research interests include integration of renewable energy resources, storage system, distribution automation system, optimized power system design and analysis.

Wei-Min Lin received his master degree in electrical engineering from National Cheng Kung University, Tainan, Taiwan, in 2007. He is currently a researcher in the Industrial Technology Research Institute, Hsinchu, Taiwan. His research interests include integration of renewable energy resources, system impact analysis, optimized power system design and analysis. 